\title{
Esterilización por Laparoscopia. Revisión de Métodos
}

\author{
Dr. Néstor H. Amorocho P.* \\ SOCIEDAD COLOMBIANA DE MEDICOS ENDOSCOPISTAS \\ PROFAMILIA - CALI 1981
}

La esterilización quirúrgica voluntaria es el método anticonceptivo más usado en el mundo.

Se calcula que aproximadamente 80 millones de parejas regulan su fecundidad en esta forma, teniendo en cuenta que en 1950 se estimaba un número global entre 3 y 4 millones, ascendiendo en 1970 a 20 millones y a 65 en 1975 (15).

La laparoscopia, inserción de un instrumento óptico en la cavidad abdominal, fue efectuada por primera vez en humanos por Jacobaeus en 1910. Inicialmente utilizada como una técnica de diagnóstico, fue propuesta como técnica de esterilización por A.T. Anderson(U.S.A.) en 1937 (1).

El invento de instrumental como endoscopios y cánulas especiales que permiten una gran variedad de planos de aproximación a las Trompas de Falopio, ha contribuido a nuevos métodos de

\footnotetext{
* Vicepresidente Sociedad Colombiana de Médicos Endoscopistas.

Laparoscopista - Profamilia -

Asociación Vallecaucana de Médicos' Endoscopistas.
}

oclusión tubaria. Como resultado, la incisión tradicional (laparatomía) ha sido remplazada por una incisión punción, muy pequeña (laparoscopia) ó por abordajes transvaginales (Colpotomia, Culdoscopía) ó transcervicales (histeroscopía), los cuales no requieren incisión.

Estas vías de abordaje permiten la oclusión de cualquier parte de las trompas de Falopio: a) Infund ibulo (extremo fimbrial de la trompa), que puede ser extirpado, quemado, obturado ó cubierto con un casquete.

b) Ampolla o Istmo (parte media del oviducto) puede ser ligada, cortada, extirpada, cauterizada o colocársele un anillo o grapa;

c) La porción intersticial del oviducto (unión útero - tubaria) puede ser coagulada o bloqueada con sustancias qu ímicas o tapones (15).

La selección de la vía de acceso y la técnica para oclusión tubaria depende del adiestramiento del médico, el grado de pericia y conocimientos, en cuanto a la seguridad y eficacia de cada uno de los métodos. Los procedimientos endoscópicos, requieren un mayor adiestramiento y pericia que las otras técnicas, 
mientras que la mayoría de los acercamientos transcervicales aún se consideran experimentales y son menos efectivos que los abordajes efectuados con otros métodos (15).

El uso de cauterio y algunas sustancias químicas están asociadas a índices mayores de lesión de estructuras adyacentes a las trompas que la ligadura, la aplicación de grapas o anillos. Los procedimientos a través del fondo del saco están asociados con una tasa de infección más alta que la de los efectuados por vía abdominal.

El potencial de reversibilidad es cada vez más importante en la selección de un método, en particular, para la oclusión tubaría. La experiencia está demostrando cada vez más, tanto en países de desarrollo como en los desarrollados, que la mayoría de las mujeres que seleccionan la esterilización prefieran un método permanente, pero muchas, particularmente los grupos de edad joven, aceptarían con agrado un medio de esterilización que pudiera ser reversible.

En Colombia los servicios de esterilización voluntaria son ofrecidos por, Profamilia (Asociación Pro-bienestar de la familia), Ascofame (Asociación Colombiana de Facultades de Medicina), muchos hospitales y médicos privados. Según datos estimados, durante 1977 se efectuaron más de 40.000 esterilizaciones, casi todas femeninas (7).

Del total de esterilizaciones para el mismo año, 28.530 fueron efectuadas por Profamilia y casi la mitad de los procedimientos (12.489) fueron realizados por laparoscopia.

\section{Laparoscopia - equipo}

Sistema óptico: Angulo de visión de $60^{\circ}$ a $180^{\circ}$. Los laparoscopios actuales tienen sistemas ópticos que permiten un efecto "Zoom": amplificación a distancias menores de $5 \mathrm{~cm}$ y reducción en distancias mayores. Distancia promedio de trabajo de 7 a $8 \mathrm{~cm}$.

FIBRO-OPTICA: Cable fibro-óptico compuesto por filamentos de $0.05 \mathrm{~mm}$ de diámetro y más de 200.000 fibras Exige manejo cuidadoso.

FUENTE DE LUZ: Lámpara parabólica cuya luz se refleja en un espejo cóncavo que dirige los haces luminosos centrados, hacia el cable fibro-óptico.

NEUMOPERITONEO: comunmente se usa C02 para obtener Neumoperitoneo. La punción se efectua generalmente en el borde inferior del ombligo. Se reduce así el riesgo de lesionar vasos grandes o herir el intestino. Se usan 1.000 a $3.000 \mathrm{ml} \mathrm{C02}$. Se utiliza una aguja de Verres (que contiene una cánula roma retráctil) o una aguja corriente de Tuohy.

TROCAR: se practica incisión transversal o longitudinal en el borde inferior del ombligo. Introducir el trocar (mandril y camisa) de manera similar a como se hizo con la aguja, ángulo de $45^{\circ}$.

\section{Laparoscopia - esterilización}

A partir de 1950, el interés por la laparoscopia trajo consigo una técnica diagnóstica y quirúrgica que viene a brindar un método inigualable al ginecólogo. En nuestra generación, desde el punto de vista diagnóstico, ha mejorado notablemente el diagnóstico pélvico en esterilidad, embarazo ectópico, endometriosis, enfermedad inflamatoria pélvica y estudio del dolor pélvico, entre otras (1) (2).

El procedimiento de esterilización femenina ha evolucionado en forma muy rápida y gracias a las innovaciones de los equipos, ha sido cambiante en los últimos 10 años. 
El método de electrofulguración por dos incisiones, técnica clásica, inicial, fue remplazado en 1971 por el de una incisión, utilizado el laparoscopio quirúrgico de Jacobs - Palmer. (1).

Aparte del uso de una o dos incisiones, las mayores diferencias en la técnica operatoria se relacionan con la extensión y el punto de acción sobre las trompas de Falopio. La opinión varía en relación a la técnica de electrocoagulación: coagular, coagular y dividir, o coagular dividir y retirar una sección de las trompas de Falopio.

La mayoría de operadores sujetan y coagulan el istmo, no obstante Frangenheim ve un riesgo a la coagulación de la trompa en el istmo, pues ahí está localizada una rama de la arteria uterina, a la cual se puede lesionar produciendo hemorragía (5).

Steptoe, antes de cortar las trompas en el istmo, coagula extensamente a lado y lado e incluye el mesosalpinz, de tal manera que los vasos que quedan por debajo de la trompa también se coagulan, con menos posibilidades de hemo rragía. Después de que se ponen blancos $2 \mathrm{~cm}$ de la trompa, corta entre las áreas cauterizadas (14).

De Boer y Dunster, dividen la trompa en el extremo lateral de la trompa coagulada (3).

Quienes defienden solamente la electrocoagulación de las trompas sostienen que hay menos hemorragía, menos traumatismo y menos posibilidad de fístula tubaria.

Jordan y colaboradores efectuaron electrocoagulación sola y electrocoagulación con división en uno dos $y$ tres puntos a lo largo de la trompa. Concluyeron en su estudio de 910 pacientes que la división de la trompa para esterili- zación por electrocoagulación no era ni necesaria ni deseable. De todos modos quienes solo hacen coagulación están en minor ía (10).

La mayoría de los médicos que coagulan y dividen las trompas, lo hacen así puesto que han notado permeabilidad tubaria persistente en algunas pacientes sometidas a electrocoagulación solamente. Steptoe informó que la mitad de $\mathbf{5 0}$ pacientes esterilizadas por electrocoagulación solamente, tenían aún permeables las trompas (13).

Liston y colaboradores ensayaron la electrocoagulación sola en 30 pacientes durante tres meses, pero regresaron a la coagulación y división pues encontraron permeabilidad tubaria persistente en el $10 \%$ de los casos.

Quienes coagulan y retiran una parte de la trompa, lo hacen casi siempre con el fin de enviar a estudio histológico la porción extirpada. Muchos de ellos informaron que pinzan la trompa de Falopio una vez, la coagulan y extirpan la porción del oviducto coagulada (16).

Obviamente las diferentes técnicas utilizadas para esterilización por electrocoagulación laparoscópica están relacionadas y dependientes de los instrumentos y equipo disponibles.

Por último, se transcribe la recomendación hecha en abril de 1975 por el grupo de expertos sobre esterilización, de la Federación Internacional de Planificación Familiar, reunido en Londres: "No se recomienda seccionar partes del conducto uterino para biopsia y examen histológico como una forma de controlar el éxito de la operación. Tal procedimiento podría aumentar el riesgo de hemorragia, hacer la reversibilidad más difícil, aumentar el trabajo de los servicios patológicos y aumentar el costo de la operación para la paciente o el servi- 
cio, todos ellos pueden evitarse ya que a pesar de ser una práctica común, el grupo de expertos no lo considera como parte esencial de la esterilización femenina (11).

\section{Grapas o Clips.}

Evans había sugerido que las grapas eran un método seguro y rápido para ocluir los oviductos, en 1953 (4). Previamente las mismas grapas eran usadas para facilitar la hemostasia durante cirugía y se había demostrado su efectividad, para este propósito.

Posteriormente, la aplicación de ganchos en las trompas de Falopio para efectos de esterilización, fue descrita por Neuman y Frick en Estados Unidos en 1960 (8).

Hulka, en la década de los años 70 , diseñó con la colaboración de Omran de la Universidad de Carolina del Norte (U.S.A.) un gancho de presión hecho de "Lexan", un plástico duro, con resorte de acero inoxidable $y$ con un cierre de silástico (8).

Aunque la grapa puede ser colocada por cualquier vía o procedimiento, el aplicador inicialmente fue diseñado para uso con laparoscopio.

En un estudio de seguimiento de 907 pacientes se registraron 24 fallas $(2.6 \%)$. Hulka sostiene que si la manufactura de las grapas es correcta y son aplicadas adecuadamente, la tasa de embarazo sería aproximadamente de 2/1.000 ó menos (9).

\section{Grapas de Tantalio}

\section{VENTAJAS PRINCIPALES}

- Simple diseño y aplicación

- Potencialmente reversible
- Económica

- Posible aplicación por distintas vías de abordaje

\section{DESVENTAJAS PRINCIPALES}

- Elevada tasa de fallas (entre 0 y 1.1 por ciento).

- Riesgo de embarazo ectópico

Grapas con resorte - Hulka

VENTAJAS PRINCIPALES

- Baja morbilidad

- Posible aplicación en pacientes ambulatorias

- Potencialmente reversible

- Eficaz (tasa de falla 0.2 a $1.5 \%$ )

\section{DESVENTAJAS PRINCIPALES}

- Alto costo de grapa y aplicador

- Técnicamente difícil

\section{Clip de Filshie}

El clip de Filshie es hecho de titanio y recubierto con Silicona, por tanto los rayos $X$ los detectan en el abdómen.

Los clips destruyen alrededor de $4 \mathrm{~mm}$ de cada trompa y la reconstrucción de los oviductos, si fuera necesaria, sugiere el inventor del clip, será más fácil después de este método de esterilización.

El aplicador laparoscópico es un instrumento para usar por segunda punción.

El clip fue desarrollado por el Dr. Marcos Filshie de la Universidad de Nottinghan, Gran Bretaña (6), quien informa que después de mejorar la estructura inicial del clip en enero de 1979, las ratas de falla serán al menos 
tan buenas como cualquiera otro método aceptable.

Actualmente se está experimentando en Profamilia - Cali, empleando técnicas de minilaparatomías y laparoscopia. Sobre una cifra de 350 casos, los resultados preliminares son alentadores.

\section{Bandas de silastic, anillo de Yoon o Anillo Falope}

En 1973, Yoon (U.S.A.), inició sus experiencias, usando un anillo de caucho de silicona. Es una pequeña rueda de $1 \mathrm{~mm}$ de diámetro interior que hace una presión entre 0,3 y 0,4 libras por $2.5 \mathrm{~cm}^{2}$ y que recupera su forma y tamaño de 90 a 100 por ciento, después de aplicado, si no se estira más de $6 \mathrm{~mm}$.

El anillo fue desarrollado para eliminar los peligros del electrocauterio durante la laparoscopia y proveer un método que sobrepasara la efectividad de las grapas de tantalio, en la oclusión tubaria (17). El equipo básico requiere de un laparoscopio especial, para técnica de una, incisión. Las tenacillas ó ganchos de este laparoscopio se abren desde el extremo, se toma la trompa y haciendo un asa se lleva a la cámara del laparoscopio. La banda de silástico se "disparará" sobre el asa o acodadura de la trompa, gracias a un sistema de camisa y émbolo (1).

La acodadura tubaria termina por ser avascular y formar un granuloma similar a la salpingitis istmica nodosa o permanece desconectadas de porción vecina de la Trompa. La destrucción de la trompa se hace secundariamente por isquemia y en consecuencia puede provocar dolor inicial (1).

\section{Bandas de silastico}

\section{VENTAJAS PRINCIPALES}

- Procedimientos en pacientes ambulatcrias (alta de 3 a 6 horas)
- Baja morbilidad

- Baja tasa de falla

- Potencialmente reversible

\section{DESVENTAJAS PRINCIPALES}

- Se requiere aplicador especial

Las tasas de embarazo, computadas para aquellas mujeres que no estaban embarazadas al momento de una esterilización y que de acuerdo con el operador, la intervención fue exitosa, en un estudio realizado por el Programa Internacional de Investigaciones de Fecundidad, en diferentes países del mundo, con un seguimiento de 12 meses fue: $0.3 \%$ para el anillo de silástico; $0,2 \%$ para fulguración y $2.1 \%$ para la grapa con resorte de Hulka (15).

Nuestra experiencia en Profamilia Cali, Colombia usando las Bandas de Silástico de Yoon, sin especificación sobre pacientes sometidas a esterilización en fase lute ínica, y considerando que es un centro de entrenamiento a nivel internacional, sobre un total de 5.535 mujeres, de enero de 1978 a mayo de 1980 da un número global de embarazo de $51(0.92 \%)$.

\section{STERILIZATION BY LAPAROSCOPY}

\section{Summary}

Any of all eligible methods of sterilization by laparoscopy must comply with at least the following basic principles:

1. The operation must be performed in no more than a four-hour hospital stay. It will be an out-patient procedure.

2. The actual duration of surgery must be less than 30 (thirty) minutes. 
3. The method should be simple enough so that any surgeon can perform the laparoscopy.

4. The procedure must yield a positive outcome as compared to other sterilization techniques, and must compete with them.

5. The overall cost of the surgical technique must be $90 \%$ less than that of a pelvic laparotomy.

6. The article presents authors experience with 5.535 laparoscopic sterilizations performed at PROFAMILIA in Cali, with a pregnancy rate of $0.92 \%$. Special considerations on reversibility are also included.

PROFAMILIA - CALI

\section{LAPAROSCOPIAS ESTERILIZACION}

TASA DE EMBARAZO

\begin{tabular}{|c|c|c|c|}
\hline Año & $\begin{array}{c}\text { No. } \\
\text { Laparoscopias }\end{array}$ & $\begin{array}{c}\text { No. } \\
\text { embarazos }\end{array}$ & Porcentaje \\
\hline 1978 & 2.456 & 36 & $0.68 \%$ \\
\hline 1979 & 2.176 & 11 & $0.50 \%$ \\
\hline 1980 & 903 & 4 & $0.44 \%$ \\
\hline TOTAL & 5.535 & 51 & $0,92 \%$ \\
\hline
\end{tabular}

\section{Consideraciones sobre reversibilidad:}

Resumen: cualquiera de los métodos elegibles para esterilización por laparoscopia, debe ajustarse a los siguientes principios básicos mínimos:

1. La operación debe efectuarse en menos de 4 horas de tiempo de hospitalización. Se hará como procedimiento extrahospitalario ó ambulatorio.
2. El tiempo quirúrgico real debe ser menos de 30 minutos.

3. El método debe ser lo bastante sencillo como para que el cirujano corriente lo pueda hacer.

4. Los resultados de la operación deben estar a la altura de las técnicas de esterilización, provisionales, y competir con ellas.

5. El costo de la técnica quirúrgica debe ser 90 por 100 menor del costo de una laparatomía pélvica.

6. Se muestra nuestra experiencia en 5.535 casos atendidos en Profamilia, Cali, con tasa de embarazo del $0.92 \%$, haciéndose consideraciones especiales sobre aspectos de reversibilidad.

En la medida en que aumenta el número de mujeres esterilizadas, crece demanda para revertir el procedimiento cuando acontecimientos no previstos como la muerte de un hijo o un nuevo matrimonio, hacen que las personas cambien de idea.

Mediante delicadas técnicas de microcirugía, algunos expertos han logrado tasas de embarazo, después de reversión de esterilización de alrededor del 60 por ciento, el doble de la tasa de hace 20 años.

Puesto que existen obstáculos para la reversión exitosa por microcirugía, debe informarse en forma muy clara a las mujeres que solicitan esterilización que esta sigue constituyendo un método permanente de control de la fecundidad.

El procedimiento quirúrgico para revertir la esterilización femenina se basa en la recanalización ó anastomosis de las porciones de las trompas que fueron dañadas o seccionadas mediante una intervención anterior. El éxito de la opera- 
ción para lograr la permeabilidad tubaria seguida de un embarazo, depende en gran parte del estado de las trompas previo a la reversión. A mayor daño de la trompa, durante la esterilización, menor probabilidad de éxito tendrá el procedimiento de reversión.

Algunas formas de esterilización son más reversibles que otras. La técnica de electrocauterización destruye $4 \mathrm{~cm} \quad 0$ más de trompa y es la más difícil de revertir.

La ligadura de Pomeroy, utilizada en el mundo entero, extirpa un promedio

\section{Referencias Bibliográficas}

1. AMOROCHO, N.H., Laparoscopia. Revisión de Temas. Revista Colombiana de Obst. y Ginec., Vol. XXX No. 5, Septiembre-octubre 1979.

2. AMOROCHO, N.H.; AMASHTA, F.; RUIZ, M.S.; Dolor Pélvico. Revista Colombiana de Obst. y Ginec., Vol. XXXI No. 6, Noviembre-Diciembre, 1980.

3. BOER, C.H. de and DUNSTER, G., Tubal Electrocoagulation under Laparoscopic Control, The Lancet 1: 997, ma yo 1970.

4. EVANS, T.N., Simplified method for sterilization of the female. American Journal of Obstetrics and Gynecology 66 (2): 393.95 August 1953.

5. FRANHENHEIM, H., Tubal División un der Endoscopic Control, German Medical Monthly 9: 421-422, 1964, English Translation.

6. FILSHIE, MARCUS., Nottinghan University. Cambridge, Gran Bretaña. Información personal, Cali, noviembre 1980. de $3 \mathrm{~cm}$ de trompa y su reversión es menos difícil.

El anillo de silástico y el clip, pueden ser lo más reversibles, aunque aún se requiere la recanalización quirúrgica.

El clip puede ser el más favorable para la reversibilidad, entre los métodos usados actualmente, puesto que daña sólo un centímetro ó menos de trompa, pero todavía existen pocos informes al respecto. Se continúan realizando investigaciones sobre técnicas de esterilización que garanticen el control de fecundidad, que produzcan un daño mínimo a las trompas y que eviten la necesidad de recanalización de los segmentos tubarios.

7. GREEN, P. CYTHIA. Esterilización voluntaria: El método anticonceptivo de mayor aceptación en el mundo. Population Reports, Series M, No. 2. Jonhns Hopkins University. Population Information Program, octubre 1978.

8. HULKA, J.F. and OMRAM, K.F., Comparative Tubal occlusion: Rigid and Spring - Loaded clips. Fertility and Sterility, 23 (9): 633-39 septiembre 1972.

9. HULKA, J.F., Spring clip Sterilization: one year Followup of 1.000 cases. Advances in female sterilization technology, Hagerstown, Maryland, Harper an Row, 1976.

10. JORDAN, J.A., EDWARDS, R. L., PEARSON, J., and MASKERY, P.L.K., Laparoscopic sterilization and Follow-Up. Hysterosalpingograms, the Journal of Obst. and Ginec. of the Bristish Common wealht 78: 440-466, mayo 1971.

11. KLEIMAN, R.L.,, Male and Female sterilization. A report of the I.P.P.F. Panel of experts on Sterilization, London, April 19-20, 1975. Second Edition London. I.P.P.F., 1975. 
12. LISTON, W.A., BRADFORD, W., DOWNIE, J., Female Sterilization by Tubal electrocoagulation Under Laparoscopic Control, The Lancet 382-83, febrero 1970.

13. STEPTOE, P.C., Problems of Laparoscopic Sterilization, The Lancet 1: 115, mayo 1972.

14. STEPTOE, P.C., Female Sterilization, Nursing Times 67: 1.529-1.530, Diciembre 1971 .

15. WORTMAN, R.N. JUDITH. Esterilización tubaria - Revisión de métodos- Popula- tion Reports Series C, No. 7. The Gecrge Washington University. Departament of medical and Public Affairs, Diciembre 1977.

16. WHEELESS, CLIFFORD R., Jr., Instrument and Method - Elimination of Second Incision in Laparoscopic Sterilization, Obst. and Gynec. 36 (2) 208-211, agosto 1970 .

17. YOON, I.B., KING, T.M., The Iaparoscope falope ring Procedure. Advances in female sterilization technology. Hagerstown, Maryland, Harper and Row, 1976. 